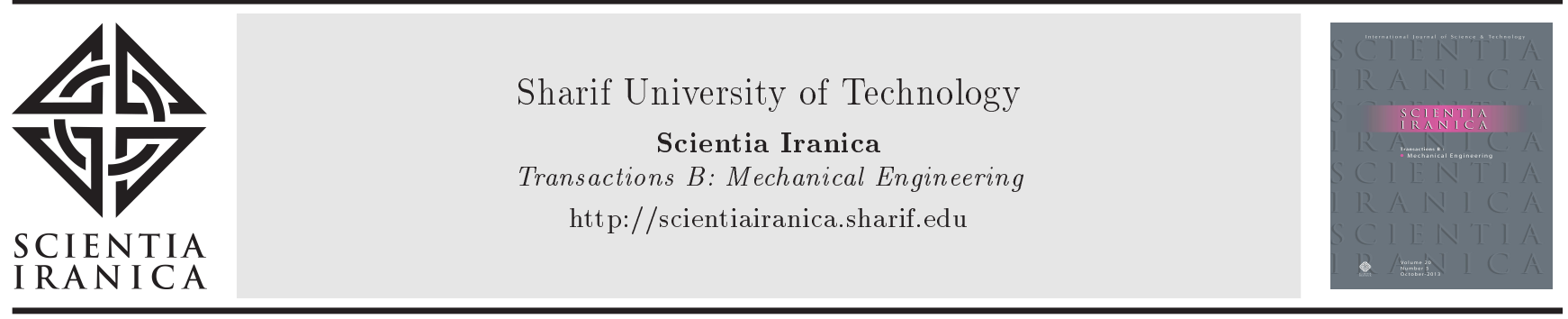

Research Note

\title{
A collocation algorithm based on quintic B-splines for the solitary wave simulation of the GRLW equation
}

\author{
H. Zeybek ${ }^{a, *}$ and S. Battal Gazi Karakoç \\ a. Department of Applied Mathematics, Faculty of Computer Science, Abdullah Gül University, 38080 Kayseri, Turkey. \\ b. Department of Mathematics, Faculty of Science and Art, Nevşehir Hacı Bektas Veli University, 50300, Nevşehir, Turkey. \\ Received 22 January 2017; received in revised form 21 January 2018; accepted 21 July 2018
}

\author{
KEYWORDS \\ GRLW equation; \\ Finite element \\ scheme; \\ Quintic B-spline; \\ Solitons; \\ Undular bore.
}

\begin{abstract}
In this article, a collocation algorithm based on quintic B-splines is proposed to find a numerical solution to the nonlinear Generalized Regularized Long Wave (GRLW) equation. Moreover, to analyze the linear stability of the numerical scheme, the vonNeumann technique is used. The numerical approach to three test examples consisting of a single solitary wave, the collision of two solitary waves, and the growth of an undular bore is discussed. The accuracy of the method is demonstrated by calculating the error in $L_{2}$ and $L_{\infty}$ norms and the conservative quantities $I_{1}, I_{2}$ and $I_{3}$. The findings are compared with those previously reported in the literature. Finally, the motion of solitary waves is graphically plotted according to different parameters.
\end{abstract}

(C) 2019 Sharif University of Technology. All rights reserved.

\section{Introduction}

The nonlinear wave phenomenon has an instrumental role in predicting natural events. The long waves in water of varying depths are modeled by equations of motion. The equations introduced for small amplitude waves are of nonlinear terms. The Regularized Long Wave (RLW) equation was initially introduced as a model for small-amplitude long waves on the surface of water in a channel by Peregrine $[1,2]$. Here, Peregrine examined the growth of an undular bore from a long wave. According to him, when the long wave of elevation travels in shallow water, it steepens and forms a bore. The RLW equation was discussed as an improved model of more common Korteweg-de Vries $(\mathrm{KdV})$ equation by Benjamin et al. [3]. The $\mathrm{KdV}$ equation defines long waves by assuming a small

\footnotetext{
*. Corresponding author.

E-mail addresses: zeybek.halil45@gmail.com (H. Zeybek); sbgkarakoc@nevsehir.edu.tr (S.Battal Gazi Karakoç)
}

wave amplitude and a large wave length in nonlinear dispersive and many other physical systems. Then, the idea of Equal Width (EW) wave equation, which has both positive and negative amplitudes with the same width, was proposed by Morrison et al. [4]. Therefore, the Generalized Regularized Long Wave (GRLW) equation and the Generalized Equal Width (GEW) wave equation offer some technical advantages over the Generalized Korteweg-de Vries (GKdV) equation. Such types of wave equations have solitary wave solutions, which are pulse-like.

The nonlinear GKdV equation has the following form:

$$
U_{t}+\varepsilon U^{p} U_{x}+\mu U_{x x x}=0 .
$$

The nonlinear GEW equation is described as follows:

$$
U_{t}+\varepsilon U^{p} U_{x}-\mu U_{x x t}=0,
$$

and the nonlinear GRLW equation, discussed here, is given by:

$$
U_{t}+U_{x}+p(p+1) U^{p} U_{x}-\mu U_{x x t}=0,
$$

subject to physical boundary conditions $U \rightarrow 0$ as 
$x \rightarrow \pm \infty$, in which subscripts $t$ and $x$ represent time and spatial differentiations, $\varepsilon$ and $p$ are the positive integers, and $\mu$ is the positive constant. The boundary and initial conditions are assumed to be as follows:

$$
\begin{aligned}
& U(a, t)=0, \quad U(b, t)=0, \quad t>0, \\
& U_{x}(a, t)=0, \quad U_{x}(b, t)=0, \quad t>0, \\
& U(x, 0)=f(x), \quad a \leq x \leq b,
\end{aligned}
$$

where $f(x)$ is the prescribed function at the interval $[a, b]$ and will be determined next. In the fluid problems, $U$ is related to the vertical displacement of the water surface or a similar physical quantity. In the plasma applications, $U$ defines the negative of the electrostatic potential. Hence, the solitary wave solution of Eqs. (1) to (3) reveals what many physical phenomena with weak nonlinearity and dispersion waves such as nonlinear transverse waves in shallow water, ion-acoustic, and magnetohydrodynamic waves in plasma and phonon packets in nonlinear crystals mean.

Indeed, the nonlinear RLW equation is formed by obtaining $p=1$ in Eq. (3). In the literature, there are large quantities of studies on the RLW equation. In the $1960 \mathrm{~s}$, Peregrine studied the RLW equation with the growth of an undular bore $[1,2]$. The approximate analytical method for the simulation of wave propagation in the nonlinear RLW equation was investigated by Morrison et al. [4]. Quadratic B-spline collocation algorithm for the nonlinear RLW equation was proposed by Raslan [5]. The RLW equation was solved numerically by using collocation algorithm based on cubic, septic, quantic, and sextic B-splines [6-9]. Galerkin finite-element method with quintic, quadratic B-splines was used to find numerical solutions of the one-dimensional RLW equation by Dağ et al. [10] and Esen and Kutluay [11]. The new Galerkin method was set up by Mei and Chen by using linear finite elements for the RLW equation [12].

In the case of $p=2$, Eq. (3) is known as the Modified Regularized Long Wave (MRLW) equation. The numerical solution to the MRLW equation was found by using a collocation method based on quintic, cubic, quartic, and septic B-splines [13-18]. Ali employed the method of mesh free to obtain a numerical solution to the MRLW equation [19]. Collocation algorithm has been newly set up with extended cubic B-splines for the numerical calculation of the MRLW equation by Dag et al. [20]. Moreover, the multi-grid method was developed for the numerical calculation of the MRLW equation by Abo Essa et al. [21].

So far, solitary wave solutions of the nonlinear GRLW equation have been found with some solution techniques by many researchers. Bona et al. [22] obtained both stable and unstable solitary-wave solutions of the nonlinear GRLW equation. Numerical methods based on finite difference scheme, He's variational iteration scheme, mesh-free technique, Petrov-Galerkin scheme, element-free approximation, and second-order compact finite difference scheme were introduced for GRLW equation [23-28]. The generalized KdV and RLW equations were solved exactly and numerically by using Adomian decomposition method [29]. Hamdi et al. [30] investigated the new exact solution approach to GRLW and its simpler alternative model, GEW equation. An approximate quasilinearization technique was designed to obtain the solitary wave solutions to nonlinear GRLW equation with an initial condition on the effects of undular bore by Ramos [31]. Mohammadi [32] obtained a numerical solution to the nonlinear GRLW equation using collocation algorithm based on exponential B-spline finite elements. Zeybek and Karakoç used a finite element method with B-splines to solve the GRLW equation [33,34]. Lately, collocation scheme based on B-spline finite elements was investigated for solving the Complex Modified Korteweg-de Vries (CMKdV), the generalized nonlinear Schrodinger (GNLS) equation, and generalized Burgers-Fisher and Burgers-Huxley equations [35-37]. Moreover, PetrovGalerkin finite element method based on B-splines was presented for the numerical calculation of the modified Korteweg-de Vries (mKdV) equation by Ak et al. [38].

Considering the numerical algorithms applied to a similar type of nonlinear equations; in this paper, we have implemented the quintic B-spline collocation approach to GRLW equation.

\section{Numerical algorithm: Quintic B-spline collocation method}

Consider a partition of the interval $[a, b]$ into $N$ equal subintervals by the points $x_{m}, m=0,1, \cdots N$ such that $h=\frac{b-a}{N}=\left(x_{m+1}-x_{m}\right)$. The set of quintic B-spline functions $\left\{\phi_{-2}(x), \phi_{-1}(x), \cdots, \phi_{N+2}(x)\right\}$ at the knots $x_{m}$ forming a basis for the functions defined over the solution region $[a, b]$ is introduced by Prenter [39] (see Eq. (5) in Box I). Each quintic B-spline, $\phi_{m}$, covers 6 elements; therefore, each finite element $\left[x_{m}, x_{m+1}\right]$ is covered by 6 splines. The numerical approximation, $U_{N}(x, t)$, is described with the quintic B-spline functions by:

$$
U_{N}(x, t)=\sum_{m=-2}^{N+2} \phi_{m}(x) \delta_{m}(t)
$$

where $\delta_{m}(t)$ is the unknown time-dependent parameter and is calculated within the boundary conditions and collocation forms. By substituting B-spline functions (5) into approximate function (6), the nodal values of $U_{m}, U_{m}^{\prime}, U_{m}^{\prime \prime}$ with regard to $\delta_{m}$ are derived as follows: 


$$
\phi_{m}(x)=\frac{1}{h^{5}} \begin{cases}\left(x-x_{m-3}\right)^{5}, & {\left[x_{m-3}, x_{m-2}\right)} \\ \left(x-x_{m-3}\right)^{5}-6\left(x-x_{m-2}\right)^{5}, & {\left[x_{m-2}, x_{m-1}\right)} \\ \left(x-x_{m-3}\right)^{5}-6\left(x-x_{m-2}\right)^{5}+15\left(x-x_{m-1}\right)^{5}, & {\left[x_{m-1}, x_{m}\right)} \\ \left(x_{m+3}-x\right)^{5}-6\left(x_{m+2}-x\right)^{5}+15\left(x_{m+1}-x\right)^{5}, & {\left[x_{m}, x_{m+1}\right)} \\ \left(x_{m+3}-x\right)^{5}-6\left(x_{m+2}-x\right)^{5}, & {\left[x_{m+1}, x_{m+2}\right)} \\ \left(x_{m+3}-x\right)^{5}, & {\left[x_{m+2}, x_{m+3}\right]} \\ 0, & \text { otherwise }\end{cases}
$$

Box I

$$
\begin{gathered}
U_{N}\left(x_{m}, t\right)=U_{m}=\delta_{m-2}+26 \delta_{m-1}+66 \delta_{m} \\
+26 \delta_{m+1}+\delta_{m+2}, \\
U_{m}^{\prime}=\frac{5}{h}\left(-\delta_{m-2}-10 \delta_{m-1}+10 \delta_{m+1}+\delta_{m+2}\right), \\
U_{m}^{\prime \prime}=\frac{20}{h^{2}}\left(\delta_{m-2}+2 \delta_{m-1}-6 \delta_{m}+2 \delta_{m+1}+\delta_{m+2}\right),
\end{gathered}
$$

and the variation of $U$ over the element $\left[x_{m}, x_{m+1}\right]$ is written by:

$$
U=\sum_{m=-2}^{N+2} \phi_{m} \delta_{m} .
$$

Using the nodal values of $U_{m}$ and their space derivatives given by Eq. (7) in Eq. (3), we get:

$$
\begin{aligned}
\left(\dot{\delta}_{m-2}\right. & \left.+26 \dot{\delta}_{m-1}+66 \dot{\delta}_{m}+26 \dot{\delta}_{m+1}+\dot{\delta}_{m+2}\right) \\
& +\frac{5}{h}\left(-\delta_{m-2}-10 \delta_{m-1}+10 \delta_{m+1}+\delta_{m+2}\right) \\
& +p(p+1) Z_{m}\left(\delta_{m-2}+26 \delta_{m-1}+66 \delta_{m}\right. \\
& \left.+26 \delta_{m+1}+\delta_{m+2}\right)-\frac{20 \mu}{h^{2}}\left(\dot{\delta}_{m-2}+2 \dot{\delta}_{m-1}\right. \\
& \left.-6 \dot{\delta}_{m}+2 \dot{\delta}_{m+1}+\dot{\delta}_{m+2}\right)=0
\end{aligned}
$$

where "' represents the derivative of time and:

$$
Z_{m}=\left(U_{m}\right)^{p-1}\left(U_{m}\right)_{x} .
$$

If the Rubin and Graves' linearization approach [40] is applied to $U^{p-1} U_{x}$, we have the following formula:

$$
\begin{aligned}
& \left(U^{p-1} U_{x}\right)^{n+1}=\left(U^{p-1}\right)^{n}\left(U_{x}\right)^{n+1} \\
& \quad+\left(U^{p-1}\right)^{n+1}\left(U_{x}\right)^{n}-\left(U^{p-1}\right)^{n}\left(U_{x}\right)^{n} .
\end{aligned}
$$

The implementation of the Crank-Nicolson formula, $\delta_{m}=\frac{1}{2}\left(\delta_{m}^{n}+\delta_{m}^{n+1}\right)$, and usual forward difference approach, $\dot{\delta}_{m}=\frac{\delta_{m}^{n+1}-\delta_{m}^{n}}{\Delta t}$, to Eq. (9) leads to the following recurrence relation:

$$
\begin{gathered}
\gamma_{1} \delta_{m-2}^{n+1}+\gamma_{2} \delta_{m-1}^{n+1}+\gamma_{3} \delta_{m}^{n+1}+\gamma_{4} \delta_{m+1}^{n+1}+\gamma_{5} \delta_{m+2}^{n+1} \\
=\gamma_{6} \delta_{m-2}^{n}+\gamma_{7} \delta_{m-1}^{n}+\gamma_{8} \delta_{m}^{n}+\gamma_{9} \delta_{m+1}^{n}+\gamma_{10} \delta_{m+2}^{n}
\end{gathered}
$$

where:

$$
\begin{aligned}
& \gamma_{1}=\left(1-K+E Z_{m}-M\right), \\
& \gamma_{2}=\left(26-10 K+26 E Z_{m}-2 M\right), \\
& \gamma_{3}=\left(66+66 E Z_{m}+6 M\right), \\
& \gamma_{4}=\left(26+10 K+26 E Z_{m}-2 M\right), \\
& \gamma_{5}=\left(1+K+E Z_{m}-M\right), \\
& \gamma_{6}=\left(1+K-E Z_{m}-M\right), \\
& \gamma_{7}=\left(26+10 K-26 E Z_{m}-2 M\right), \\
& \gamma_{8}=\left(66-66 E Z_{m}+6 M\right), \\
& \gamma_{9}=\left(26-10 K-26 E Z_{m}-2 M\right), \\
& \gamma_{10}=\left(1-K-E Z_{m}-M\right), \\
& m=0,1, \cdots, N, \quad K=\frac{5 \Delta t}{2 h}, \quad M=\frac{20 \mu}{h^{2}} . \\
& E=\frac{p(p+1) \Delta t}{2}, \quad M \quad, \quad M \quad
\end{aligned}
$$

The recurrence relation (11) comprises $(N+1)$ linear equations, whereas this system involves $(N+5)$ unknowns $\left(\delta_{-2}, \delta_{-1}, \cdots, \delta_{N+1}, \delta_{N+2}\right)^{T}$. Using the boundary conditions given by Eq. (4), we delete $\delta_{-2}, \delta_{-1}$ and $\delta_{N+1}, \delta_{N+2}$ from Systems (11). In this case, the penta-diagonal matrix system can be easily achieved as follows:

$$
A \mathbf{d}^{\mathbf{n}+\mathbf{1}}=B \mathbf{d}^{\mathbf{n}}
$$

which can be solved through the penta-diagonal system. To obtain better numerical results at each time step, two or three inner iterations $\delta^{n *}=\delta^{n}+\frac{1}{2}\left(\delta^{n}-\right.$ $\left.\delta^{n-1}\right)$ are applied to $Z_{m}$. 
In order to start the iteration, initial parameters $d^{0}$ must be computed by using the following conditions:

$$
\begin{array}{ll}
U_{N}(x, 0)=U\left(x_{m}, 0\right), & m=0,1,2, \cdots, N, \\
\left(U_{N}\right)_{x}(a, 0)=0, & \left(U_{N}\right)_{x}(b, 0)=0, \\
\left(U_{N}\right)_{x x}(a, 0)=0, & \left(U_{N}\right)_{x x}(b, 0)=0 .
\end{array}
$$

Therefore, we have the ratio of the matrix equation to the initial vector $d^{0}$ :

$$
W d^{0}=b,
$$

where:

$$
W=\left[\begin{array}{cccccccc}
54 & 60 & 6 & & & & & \\
25.25 & 67.5 & 26.25 & 1 & & & & \\
1 & 26 & 66 & 26 & 1 & & & \\
& & \ddots & \ddots & \ddots & \ddots & & \\
& & & 1 & 26 & 66 & 26 & 1 \\
& & & & 1 & 26.25 & 67.5 & 25.25 \\
& & & & & 6 & 60 & 54
\end{array}\right]
$$

$d^{0}=\left(\delta_{0}, \delta_{1}, \delta_{2}, \cdots, \delta_{N-2}, \delta_{N-1}, \delta_{N}\right)^{T}$,

$$
b=\left(U\left(x_{0}, 0\right), U\left(x_{1}, 0\right), \cdots, U\left(x_{N-1}, 0\right), U\left(x_{N}, 0\right)\right)^{T} .
$$

\subsection{The solution with penta-diagonal algorithm}

Designed in the Fortran program, the solution method with penta-diagonal algorithm is given as follows: The penta-diagonal system can be written as follows:

$$
\begin{aligned}
& a_{i} \delta_{i-2}+b_{i} \delta_{i-1}+c_{i} \delta_{i}+d_{i} \delta_{i+1}+e_{i} \delta_{i+2}=f_{i}, \\
& i=0,1, \cdots, N .
\end{aligned}
$$

Firstly, the parameters are established with:

$$
\begin{aligned}
& \alpha_{0}=0, \quad \beta_{0}=c_{0}, \quad \mu_{0}=\frac{d_{0}}{\beta_{0}}, \quad \zeta_{0}=\frac{e_{0}}{\beta_{0}}, \\
& \lambda_{0}=\frac{f_{0}}{\beta_{0}}, \quad \alpha_{1}=b_{0}, \quad \beta_{1}=c_{1}-\alpha_{1} \mu_{0}, \\
& \mu_{1}=\frac{d_{1}-\alpha_{1} \zeta_{0}}{\beta_{1}}, \quad \zeta_{1}=\frac{e_{1}}{\beta_{1}}, \quad \lambda_{1}=\frac{f_{1}-\alpha_{1} \lambda_{0}}{\beta_{1}} .
\end{aligned}
$$

Afterwards, the following parameters are computed:

$$
\begin{aligned}
& \alpha_{i}=b_{i-1}-a_{i-2} \mu_{i-2}, \quad \beta_{i}=c_{i}-\alpha_{i} \mu_{i-1}-a_{i-2} \zeta_{i-2}, \\
& \mu_{i}=\frac{d_{i}-\alpha_{i} \zeta_{i-1}}{\beta_{i}}, \quad \zeta_{i}=\frac{e_{i}}{\beta_{i}}, \\
& \lambda_{i}=\frac{f_{i}-\alpha_{i} \lambda_{i-1}-a_{i-2} \lambda_{i-2}}{\beta_{i}}, \\
& \text { for } \quad i=2,3, \cdots, N .
\end{aligned}
$$

Now, the solution is obtained as follows:

$$
\begin{aligned}
& \delta_{i}=\lambda_{i}-\zeta_{i} \delta_{i+2}-\mu_{i} \delta_{i+1}, \\
& \quad i=0,1, \cdots, N-3, N-2, \\
& \delta_{N-1}=\lambda_{N-1}-\mu_{N-1} \delta_{N}, \quad \delta_{N}=\lambda_{N} .
\end{aligned}
$$

\subsection{Stability of the scheme}

To ensure the stability of the scheme, the von-Neumann technique is followed. Moreover, $U^{p}$ in the nonlinear term is considered to be locally constant. If the same steps in the presented numerical algorithm are performed, the following recurrence relation is obtained:

$$
\begin{gathered}
\alpha_{1} \delta_{m-2}^{n+1}+\alpha_{2} \delta_{m-1}^{n+1}+\alpha_{3} \delta_{m}^{n+1}+\alpha_{4} \delta_{m+1}^{n+1}+\alpha_{5} \delta_{m+2}^{n+1} \\
=\alpha_{5} \delta_{m-2}^{n}+\alpha_{4} \delta_{m-1}^{n}+\alpha_{3} \delta_{m}^{n}+\alpha_{2} \delta_{m+1}^{n}+\alpha_{1} \delta_{m+2}^{n},
\end{gathered}
$$

where:

$$
\begin{aligned}
& \alpha_{1}=\left(1-K-K E Z_{m}-M\right), \\
& \alpha_{2}=\left(26-10 K-10 K E Z_{m}-2 M\right), \\
& \alpha_{3}=(66+6 M), \\
& \alpha_{4}=\left(26+10 K+10 K E Z_{m}-2 M\right), \\
& \alpha_{5}=\left(1+K+K E Z_{m}-M\right), \quad m=0,1, \cdots, N, \\
& K=\frac{5 \Delta t}{2 h}, \quad E=\frac{p(p+1) \Delta t}{2}, \quad M=\frac{20 \mu}{h^{2}} .
\end{aligned}
$$

Then, the Fourier mode $\delta_{m}^{n}=\xi^{n} e^{i m k h}$, where $i=\sqrt{-1}$, $h$ is the step length, and $k$ is the mode number in the above equation, which produces the following equality:

$$
\begin{gathered}
\alpha_{1} \xi^{n+1} e^{i(m-2) k h}+\alpha_{2} \xi^{n+1} e^{i(m-1) k h}+\alpha_{3} \xi^{n+1} e^{i m k h} \\
+\alpha_{4} \xi^{n+1} e^{i(m+1) k h}+\alpha_{5} \xi^{n+1} e^{i(m+2) k h} \\
=\alpha_{5} \xi^{n} e^{i(m-2) k h}+\alpha_{4} \xi^{n} e^{i(m-1) k h}+\alpha_{3} \xi^{n} e^{i m k h} \\
+\alpha_{2} \xi^{n} e^{i(m+1) k h}+\alpha_{1} \xi^{n} e^{i(m+2) k h}
\end{gathered}
$$

Implementing Euler's formula $\left(e^{i k h}=\cos (k h)+\right.$ $i \sin (k h))$, we get:

$$
\xi=\frac{a-i b}{a+i b}
$$

in which:

$$
\begin{aligned}
& a=\alpha_{3}+\left(\alpha_{4}+\alpha_{2}\right) \cos [h k]+\left(\alpha_{5}+\alpha_{1}\right) \cos [2 h k], \\
& b=\left(\alpha_{4}-\alpha_{2}\right) \sin [h k]+\left(\alpha_{5}-\alpha_{1}\right) \sin [2 h k] .
\end{aligned}
$$

The modulus of $|\xi|$ is 1 , making the linearized scheme unconditionally stable. 


\section{Numerical examples and results}

In this part, the numerical approach is implemented on three examples containing a single solitary wave, the collision of two solitary waves, and the growth of an undular bore. The error in $L_{2}$ and $L_{\infty}$ norms is computed to check the efficiency and accuracy of the numerical scheme. To this end, the exact solution of GRLW equation given in Eq. (17) and the following formulas are used:

$$
\begin{aligned}
& L_{2}=\left\|U^{\text {exact }}-U_{N}\right\|_{2} \simeq \sqrt{h \sum_{J=0}^{N}\left|U_{j}^{\text {exact }}-\left(U_{N}\right)_{j}\right|^{2},} \\
& L_{\infty}=\left\|U^{\text {exact }}-U_{N}\right\|_{\infty} \simeq \max _{j}\left|U_{j}^{\text {exact }}-\left(U_{N}\right)_{j}\right| .
\end{aligned}
$$

The papers $[13,25]$ presented the exact solution to GRLW equation as follows:

$$
\begin{aligned}
& U(x, t) \\
& \quad=\sqrt[p]{\frac{v(p+2)}{2 p} \sec h^{2}\left[\frac{p}{2} \sqrt{\frac{v}{\mu(v+1)}}\left(x-(v+1) t-x_{0}\right)\right]},
\end{aligned}
$$

where $v+1$ is the velocity of the wave in the direction of propagation, $x_{0}$ is the arbitrary constant, and $\sqrt[p]{\frac{v(p+2)}{2 p}}$ defines amplitude. In addition, to register that the numerical scheme retains the physical quantities, the changes of the invariants related to mass, momentum, and energy are studied.

$$
\begin{aligned}
& I_{1}=\int_{a}^{b} U d x, \quad I_{2}=\int_{a}^{b}\left[U^{2}+\mu\left(U_{x}\right)^{2}\right] d x, \\
& I_{3}=\int_{a}^{b}\left[U^{4}-\mu\left(U_{x}\right)^{2}\right] d x .
\end{aligned}
$$

\subsection{Example 1: A single solitary wave}

The first test example is established with the initial condition of $t=0$ in Eq. (17). In order to achieve uniform and comparable numerical results, the papers $[10,13,15,17,19,25,31]$ are followed. The same values of $x \in[0,100], \mu=1$, and $x_{0}=40$ and different values of space step $h$, time step $\Delta t, p$, and $v$ are chosen. The experiments are carried out up to $t=20$.

In the first case, we consider $h=0.2,0.1, \Delta t=$

\begin{tabular}{|c|c|c|c|c|c|c|c|c|c|c|}
\hline$\|p=2\|$ & \multicolumn{2}{|c|}{$I_{1}$} & \multicolumn{2}{|c|}{$I_{2}$} & \multicolumn{2}{|c|}{$I_{3}$} & \multicolumn{2}{|c|}{$L_{2} \times 10^{4}$} & \multicolumn{2}{|c|}{$L_{\infty} \times 10^{4}$} \\
\hline Time & $v=0.1$ & $v=0.3$ & $v=0.1$ & $v=0.3$ & $v=0.1$ & $v=0.3$ & $v=0.1$ & $v=0.3$ & $v=0.1$ & $v=0.3$ \\
\hline 0 & 3.29490 & 3.58195 & 0.68342 & 1.34507 & 0.02412 & 0.15372 & 0.000 & 0.000 & 0.000 & 0.000 \\
\hline 5 & 3.29492 & 3.58195 & 0.68342 & 1.34507 & 0.02412 & 0.15372 & 0.040 & 0.095 & 0.029 & 0.051 \\
\hline 10 & 3.29493 & 3.58195 & 0.68342 & 1.34507 & 0.02412 & 0.15372 & 0.075 & 0.159 & 0.035 & 0.076 \\
\hline 15 & 3.29494 & 3.58195 & 0.68342 & 1.34506 & 0.02412 & 0.15372 & 0.101 & 0.207 & 0.036 & 0.095 \\
\hline 20 & 3.29493 & 3.58195 & 0.68342 & 1.34506 & 0.02412 & 0.15372 & 0.120 & 0.376 & 0.066 & 0.175 \\
\hline$\|p=3\|$ & \multicolumn{2}{|c|}{$I_{1}$} & \multicolumn{2}{|c|}{$I_{2}$} & \multicolumn{2}{|c|}{$I_{3}$} & \multicolumn{2}{|c|}{$L_{2} \times 10^{4}$} & \multicolumn{2}{|c|}{$L_{\infty} \times 10^{4}$} \\
\hline Time & $v=0.1$ & $v=0.3$ & $v=0.1$ & $v=0.3$ & $v=0.1$ & $v=0.3$ & $v=0.1$ & $v=0.3$ & $v=0.1$ & $v=0.3$ \\
\hline 0 & 4.06256 & 3.67753 & 1.13387 & 1.56573 & 0.09289 & 0.22683 & 0.000 & 0.000 & 0.000 & 0.000 \\
\hline 5 & 4.06258 & 3.67753 & 1.13387 & 1.56573 & 0.09289 & 0.22683 & 0.048 & 0.217 & 0.032 & 0.121 \\
\hline 10 & 4.06260 & 3.67753 & 1.13387 & 1.56573 & 0.09289 & 0.22684 & 0.088 & 0.400 & 0.038 & 0.203 \\
\hline 15 & 4.06261 & 3.67753 & 1.13387 & 1.56573 & 0.09289 & 0.22684 & 0.116 & 0.581 & 0.039 & 0.284 \\
\hline 20 & 4.06260 & 3.67753 & 1.13386 & 1.56572 & 0.09289 & 0.22684 & 0.137 & 0.918 & 0.073 & 0.438 \\
\hline$\|p=4\|$ & \multicolumn{2}{|c|}{$I_{1}$} & \multicolumn{2}{|c|}{$I_{2}$} & \multicolumn{2}{|c|}{$I_{3}$} & \multicolumn{2}{|c|}{$L_{2} \times 10^{4}$} & \multicolumn{2}{|c|}{$L_{\infty} \times 10^{4}$} \\
\hline Time & $v=0.1$ & $v=0.3$ & $v=0.1$ & $v=0.3$ & $v=0.1$ & $v=0.3$ & $v=0.1$ & $v=0.3$ & $v=0.1$ & $v=0.3$ \\
\hline 0 & 4.55093 & 3.75921 & 1.49159 & 1.72999 & 0.18389 & 0.28940 & 0.000 & 0.000 & 0.000 & 0.000 \\
\hline 5 & 4.55095 & 3.75921 & 1.49159 & 1.72999 & 0.18389 & 0.28941 & 0.059 & 0.402 & 0.034 & 0.231 \\
\hline 10 & 4.55097 & 3.75921 & 1.49159 & 1.72998 & 0.18389 & 0.28941 & 0.106 & 0.803 & 0.041 & 0.421 \\
\hline 15 & 4.55098 & 3.75921 & 1.49159 & 1.72998 & 0.18389 & 0.28941 & 0.142 & 1.235 & 0.042 & 0.627 \\
\hline 20 & 4.55097 & 3.75921 & 1.49159 & 1.72998 & 0.18389 & 0.28941 & 0.176 & 1.868 & 0.078 & 0.915 \\
\hline
\end{tabular}
0.01 , and $v=0.1,0.3$. Three invariants and errors are presented in Tables 1 and 2. It is observed from the tables that the changes of three invariants from their initial state are less than $0.03 \%$ in all computer runs.

Table 1. Invariants and errors for Example 1 when $x \in[0,100], h=0.2$, and $\Delta t=0.01$. 
Table 2. Invariants and errors for Example 1 when $x \in[0,100], h=0.1$, and $\Delta t=0.01$.

\begin{tabular}{|c|c|c|c|c|c|c|c|c|c|c|}
\hline$\|p=6\|$ & \multicolumn{2}{|c|}{$I_{1}$} & \multicolumn{2}{|c|}{$I_{2}$} & \multicolumn{2}{|c|}{$I_{3}$} & \multicolumn{2}{|c|}{$L_{2} \times 10^{4}$} & \multicolumn{2}{|c|}{$L_{\infty} \times 10^{4}$} \\
\hline Time & $v=0.1$ & $v=0.3$ & $v=0.1$ & $v=0.3$ & $v=0.1$ & $v=0.3$ & $v=0.1$ & $v=0.3$ & $v=0.1$ & $v=0.3$ \\
\hline 0 & 5.12921 & 3.86622 & 1.98857 & 1.94334 & 0.36740 & 0.37760 & 0.000 & 0.000 & 0.000 & 0.000 \\
\hline 5 & 5.12924 & 3.86622 & 1.98857 & 1.94334 & 0.36740 & 0.37760 & 0.236 & 0.752 & 0.092 & 0.402 \\
\hline 10 & 5.12926 & 3.86622 & 1.98857 & 1.94334 & 0.36740 & 0.37760 & 0.458 & 1.554 & 0.179 & 0.823 \\
\hline 15 & 5.12927 & 3.86622 & 1.98857 & 1.94333 & 0.36740 & 0.37760 & 0.661 & 2.429 & 0.259 & 1.282 \\
\hline 20 & 5.12926 & 3.86622 & 1.98857 & 1.94333 & 0.36740 & 0.37760 & 0.848 & 3.390 & 0.333 & 1.785 \\
\hline$\|p=8\|$ & \multicolumn{2}{|c|}{$I_{1}$} & \multicolumn{2}{|c|}{$I_{2}$} & \multicolumn{2}{|c|}{$I_{3}$} & \multicolumn{2}{|c|}{$L_{2} \times 10^{4}$} & \multicolumn{2}{|c|}{$L_{\infty} \times 10^{4}$} \\
\hline Time & $v=0.1$ & $v=0.3$ & $v=0.1$ & $v=0.3$ & $v=0.1$ & $v=0.3$ & $v=0.1$ & $v=0.3$ & $v=0.1$ & $v=0.3$ \\
\hline 0 & 5.45779 & 3.92982 & 2.30588 & 2.07217 & 0.51946 & 0.43167 & 0.000 & 0.000 & 0.000 & 0.000 \\
\hline 5 & 5.45781 & 3.92982 & 2.30589 & 2.07217 & 0.51946 & 0.43167 & 0.268 & 1.204 & 0.108 & 0.690 \\
\hline 10 & 5.45783 & 3.92981 & 2.30589 & 2.07216 & 0.51946 & 0.43168 & 0.499 & 3.012 & 0.200 & 1.699 \\
\hline 15 & 5.45785 & 3.92981 & 2.30589 & 2.07214 & 0.51946 & 0.43170 & 0.686 & 5.690 & 0.273 & 3.184 \\
\hline 20 & 5.45784 & 3.92980 & 2.30589 & 2.07212 & 0.51946 & 0.43172 & 0.822 & 9.520 & 0.322 & 5.296 \\
\hline$\|p=10\|$ & \multicolumn{2}{|c|}{$I_{1}$} & \multicolumn{2}{|c|}{$I_{2}$} & \multicolumn{2}{|c|}{$I_{3}$} & \multicolumn{2}{|c|}{$L_{2} \times 10^{4}$} & \multicolumn{2}{|c|}{$L_{\infty} \times 10^{4}$} \\
\hline Time & $v=0.1$ & $v=0.3$ & $v=0.1$ & $v=0.3$ & $v=0.1$ & $v=0.3$ & $v=0.1$ & $v=0.3$ & $v=0.1$ & $v=0.3$ \\
\hline 0 & 5.66906 & 3.97136 & 2.52266 & 2.15744 & 0.63820 & 0.46614 & 0.000 & 0.000 & 0.000 & 0.000 \\
\hline 5 & 5.66908 & 3.97134 & 2.52266 & 2.15742 & 0.63819 & 0.46615 & 0.297 & 2.271 & 0.124 & 1.380 \\
\hline 10 & 5.66910 & 3.97133 & 2.52266 & 2.15737 & 0.63819 & 0.46620 & 0.536 & 7.775 & 0.220 & 4.595 \\
\hline 15 & 5.66912 & 3.97131 & 2.52267 & 2.15729 & 0.63819 & 0.46629 & 0.700 & 19.017 & 0.280 & 11.082 \\
\hline 20 & 5.66911 & 3.97129 & 2.52267 & 2.15714 & 0.63819 & 0.46643 & 0.764 & 39.763 & 0.288 & 22.983 \\
\hline
\end{tabular}

Table 3. Errors for Example 1 when $x \in[0,100], \mu=1$, and $t=20$.

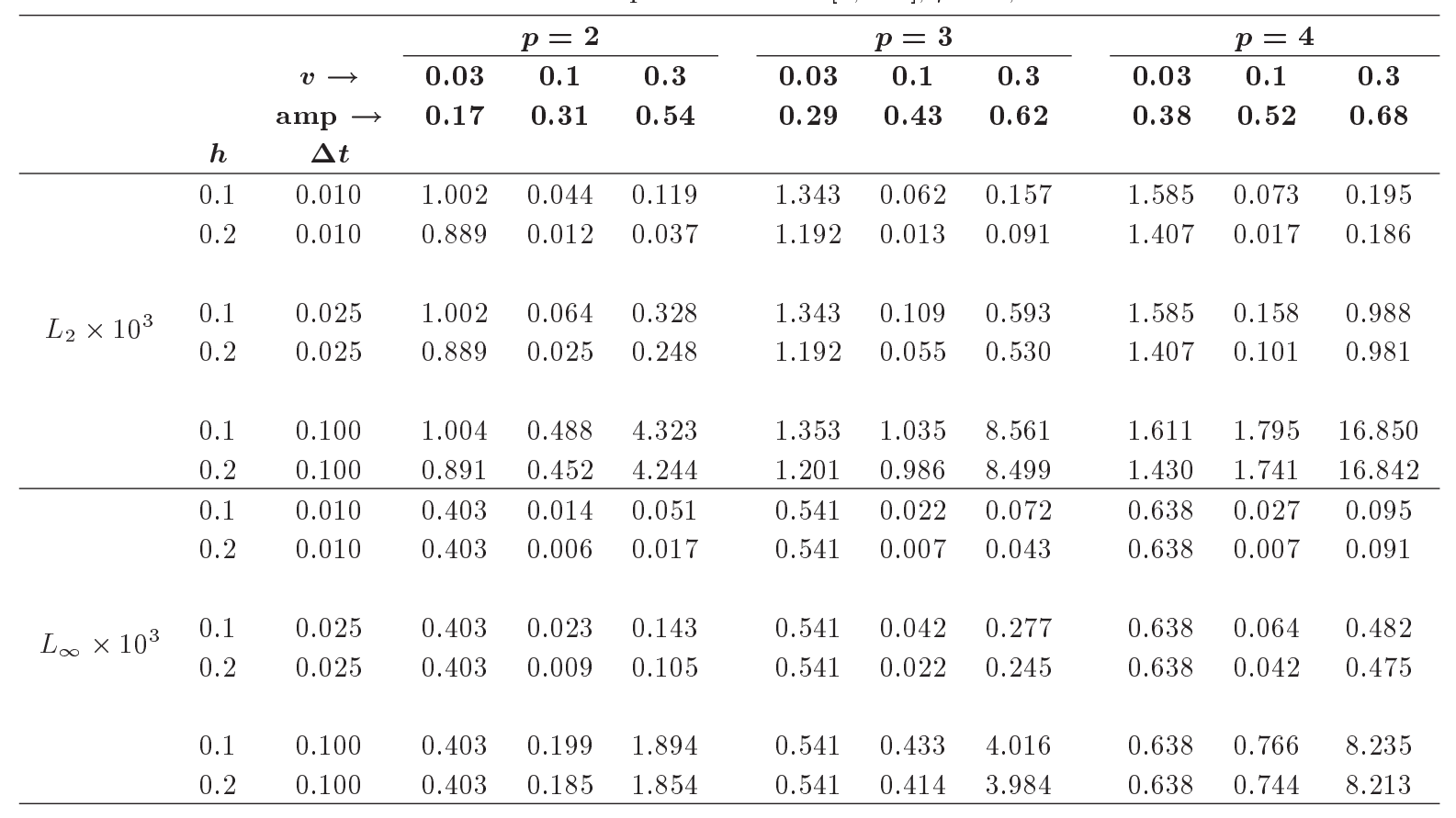

Moreover, it is found that the magnitude of $L_{2}$ and $L_{\infty}$ error norms is adequately small with increasing $p$, time, and velocity, as expected.

Second, this study seeks to examine the quantity of error norms at different velocities, space steps, and time steps. For this purpose, we have taken $h=$ $0.1,0.2, \Delta t=0.01,0.025,0.1$, and $v=0.03,0.1,0.3$. The values of $L_{2}$ and $L_{\infty}$ error norms are listed at $t=20$ in Tables 3 and 4 . In these tables, error norms are found to be small enough, and $L_{\infty}$ error is always 


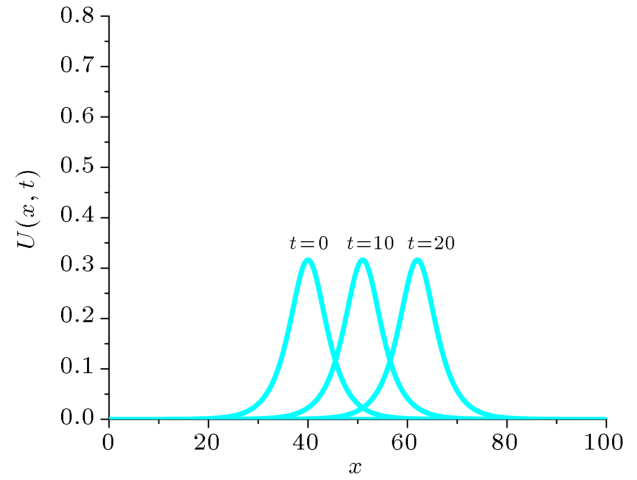

(a) $p=2$

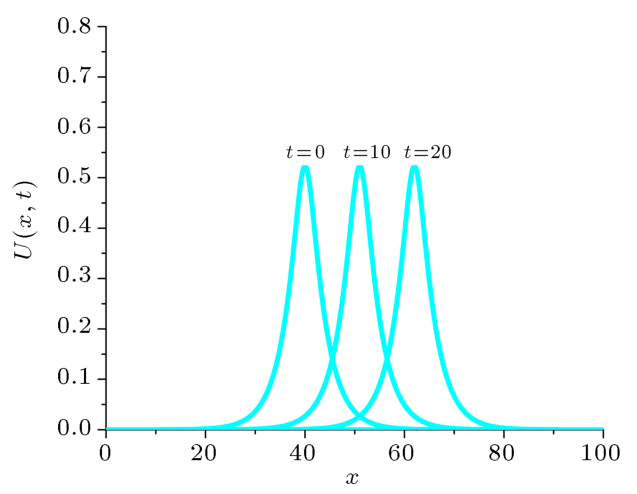

(c) $p=4$

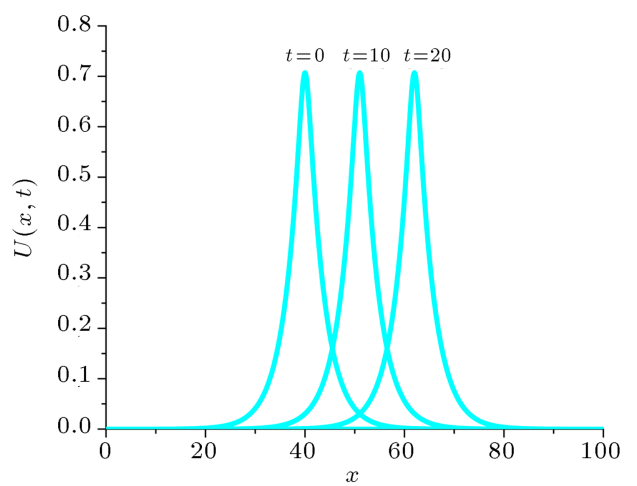

(e) $p=8$

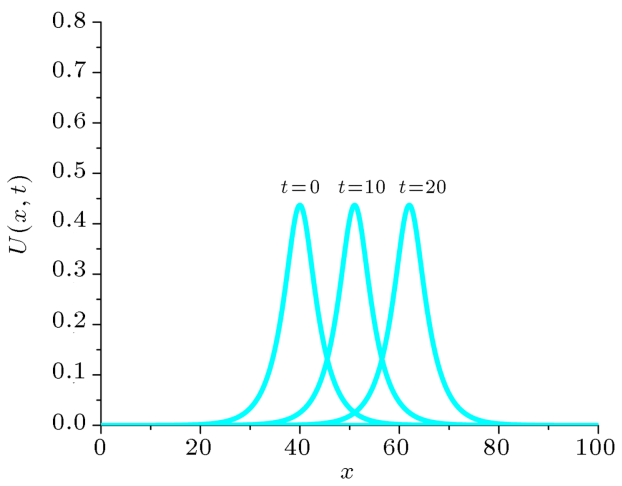

(b) $p=3$

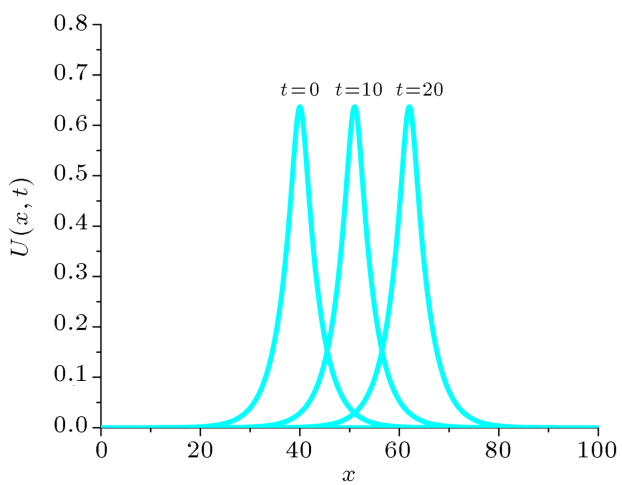

(d) $p=6$

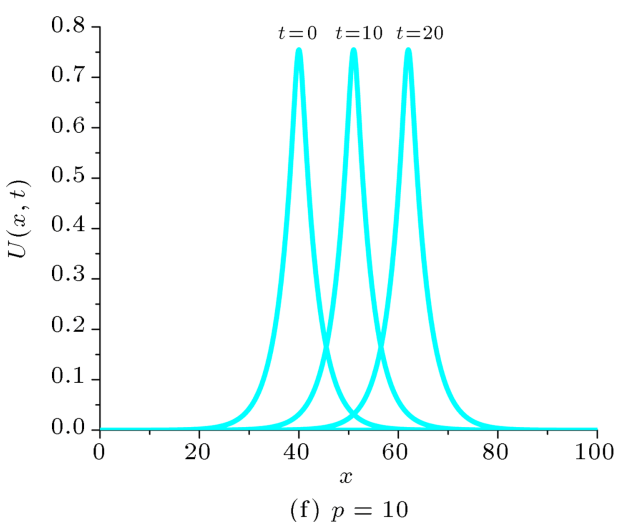

Figure 1. The motion of a single solitary wave when $x \in[0,100], v=0.1$, and $x_{0}=40$.

separated solitary waves with different amplitudes

$$
\begin{aligned}
& U(x, 0) \\
& \quad=\sum_{i=1}^{2} \sqrt[p]{\frac{v_{i}(p+2)}{2 p} \sec h^{2}\left[\frac{p}{2} \sqrt{\frac{v_{i}}{\mu\left(v_{i}+1\right)}}\left(x-x_{i}\right)\right]},
\end{aligned}
$$

where $v_{i}$ and $x_{i}, i=1,2$, are arbitrary constants.

Numerical calculation is carried out under the following conditions: $p=2, x \in[0,250], h=0.2$, $\Delta t=0.025, \mu=1, v_{1}=4, v_{2}=1, x_{1}=25, x_{2}=55$, $p=3, x \in[0,120], h=0.1, \Delta t=0.01, \mu=1$, $v_{1}=48 / 5, v_{2}=6 / 5, x_{1}=20, x_{2}=50, p=4$, $x \in[0,200], h=0.125, \Delta t=0.01, \mu=1, v_{1}=64 / 3$, $v_{2}=4 / 3, x_{1}=20$, and $x_{2}=80$. The computational data are recorded in Tables 6 and 7 , which denote that the quantities of the invariants change a little from their initial count, which are compatible with the results of the referenced paper [25]. The motion of two solitary waves is depicted at different time steps in Figures 2 and 3. As seen in these figures, at time zero, the solitary wave with larger energy is behind the second wave involving smaller energy. According to the solitary wave theory, greater energy means more velocity. Hence, over time, the large wave attains a smaller one and interposition takes place. Similarly, a wave with larger energy leaves behind the second wave with smaller energy, and the same is reiterated. 
Table 6. Invariants for Example 2 when $p=2, x \in[0,250], h=0.2, \Delta t=0.025, \mu=1, v_{1}=4, v_{2}=1, x_{1}=25$, and $x_{2}=55$.

\begin{tabular}{ccccccccc}
\hline & \multicolumn{2}{c}{$\boldsymbol{I}_{\mathbf{1}}$} & & \multicolumn{2}{c}{$\boldsymbol{I}_{\mathbf{2}}$} & & \multicolumn{2}{c}{$\boldsymbol{I}_{\mathbf{3}}$} \\
\cline { 2 - 3 } \cline { 8 - 9 } Time & QBSC & QBSPG & & QBSC & QBSPG & & QBSC & QBSPG \\
$\downarrow$ & Ours & {$[\mathbf{2 5}]$} & & Ours & {$[\mathbf{2 5}]$} & & Ours & {$[\mathbf{2 5}]$} \\
\hline 0 & 11.4676 & 11.4677 & & 14.6292 & 14.6286 & & 22.8803 & 22.8788 \\
4 & 11.4676 & 11.4677 & & 14.6277 & 14.6292 & & 22.8818 & 22.8811 \\
8 & 11.4668 & 11.4677 & & 14.1399 & 14.6229 & & 23.3695 & 22.8798 \\
12 & 11.4676 & 11.4677 & & 14.6803 & 14.6299 & & 22.8292 & 22.8803 \\
16 & 11.4676 & 11.4677 & & 14.6442 & 14.6295 & & 22.8653 & 22.8805 \\
20 & 11.4676 & 11.4677 & & 14.6309 & 14.6299 & & 22.8786 & 22.8806 \\
\hline
\end{tabular}

Table 7. Invariants for Example 2.

\begin{tabular}{ccccccccc}
\hline \multicolumn{2}{c}{ Time } & $\mathbf{0}$ & $\mathbf{1}$ & $\mathbf{2}$ & $\mathbf{3}$ & $\mathbf{4}$ & $\mathbf{5}$ & $\mathbf{6}$ \\
\hline \multirow{4}{*}{$p=3$} & $I_{1}$ & 9.6907 & 9.6894 & 9.6881 & 9.6851 & 9.6860 & 9.6848 & 9.6835 \\
& $I_{2}$ & 12.9443 & 12.9433 & 12.9391 & 12.3044 & 12.9704 & 13.0539 & 13.0028 \\
& $I_{3}$ & 17.0186 & 17.0197 & 17.0239 & 17.6586 & 16.9926 & 16.9091 & 16.9601 \\
& & & & & & & & \\
\multirow{2}{*}{$p=4$} & $I_{1}$ & 8.8342 & 8.6650 & 8.5662 & 8.4965 & 8.4529 & 8.4089 & 8.3702 \\
& $I_{2}$ & 12.1708 & 11.9332 & 11.7919 & 11.6913 & 11.4644 & 11.7254 & 11.5990 \\
& $I_{3}$ & 14.0294 & 14.2670 & 14.4083 & 14.5090 & 14.7358 & 14.4748 & 14.6012 \\
\hline
\end{tabular}

Table 8. Physical quantities for Example 3 when $x \in[-36,300], x_{0}=0, h=0.1, \Delta t=0.1, \mu=1 / 6, d=5$, and $U_{0}=0.1$.

\begin{tabular}{|c|c|c|c|c|c|c|c|c|c|}
\hline \multirow{2}{*}{$\begin{array}{c}\text { Time } \\
\downarrow\end{array}$} & \multicolumn{3}{|c|}{$I_{1}$} & \multicolumn{3}{|c|}{$I_{2}$} & \multicolumn{3}{|c|}{$I_{3}$} \\
\hline & $p=2$ & $p=3$ & $p=4$ & $p=2$ & $p=3$ & $p=4$ & $p=2$ & $p=3$ & $p=4$ \\
\hline 0 & 3.6049 & 3.6049 & 3.6049 & 0.3372 & 0.3372 & 0.3372 & 0.0014 & 0.0014 & 0.0014 \\
\hline 50 & 7.0244 & 6.9980 & 6.9954 & 0.5694 & 0.5668 & 0.5665 & 0.0041 & 0.0041 & 0.0041 \\
\hline 100 & 10.3873 & 10.3343 & 10.3290 & 0.7946 & 0.7893 & 0.7888 & 0.0051 & 0.0051 & 0.0051 \\
\hline 150 & 13.7503 & 13.6706 & 13.6626 & 1.0198 & 1.0119 & 1.0110 & 0.0061 & 0.0061 & 0.0061 \\
\hline 200 & 17.1133 & 17.0069 & 16.9961 & 1.2450 & 1.2344 & 1.2333 & 0.0071 & 0.0071 & 0.0071 \\
\hline
\end{tabular}

\subsection{Example 3: Undular bore}

Finally, we have worked on the growth of an undular bore:

$$
U(x, 0)=\frac{1}{2} U_{0}\left[1-\tanh \left(\frac{x-x_{c}}{d}\right)\right],
$$

which reflects the elevation of the water surface above the equilibrium point. The change in the water level of magnitude Eq. (20) is centered on $x=x_{c}$. To be consistent with the papers $[1,11,12]$, the parameters $U_{0}=0.1, \mu=1 / 6, h=0.1, \Delta t=0.1, x_{c}=0$, $d=5$, and $x \in[-36,300]$ are used. The three conservation laws are given in Table 8. From this table, it was observed that the change in the invariants was reasonably small. The undulation profiles at different time steps are drawn in Figures 4 to 6 . It can be concluded that the number of undulations increases when the value of $x$ rises and waves move like this for a time. Afterwards, undulations take the peak position and disappear.

\section{Conclusion}

A collocation method based on quintic B-splines was 


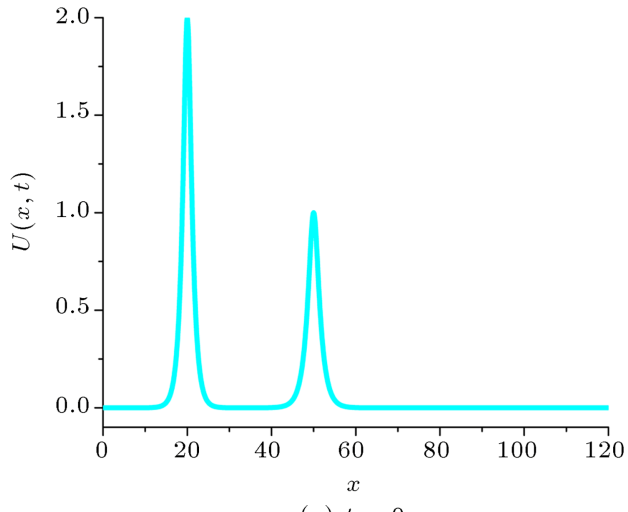

(a) $t=0$

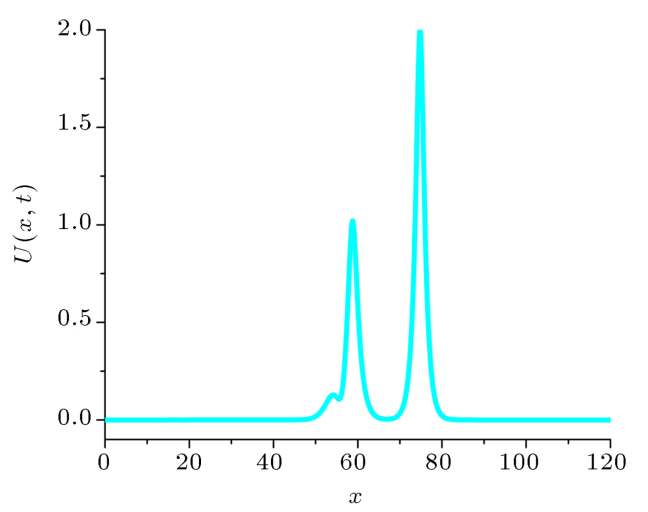

(c) $t=5$

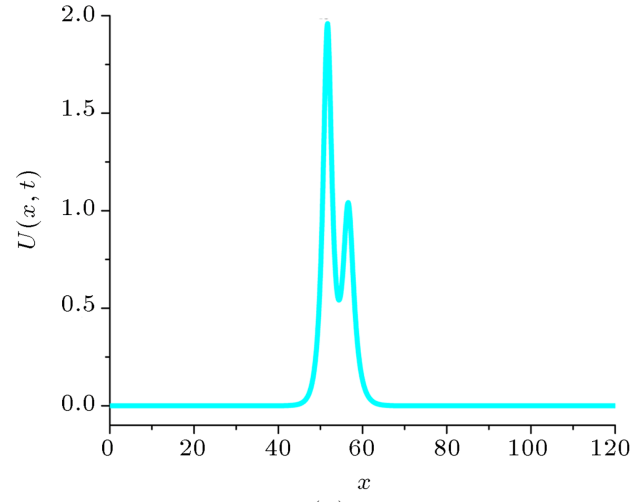

(b) $t=3$

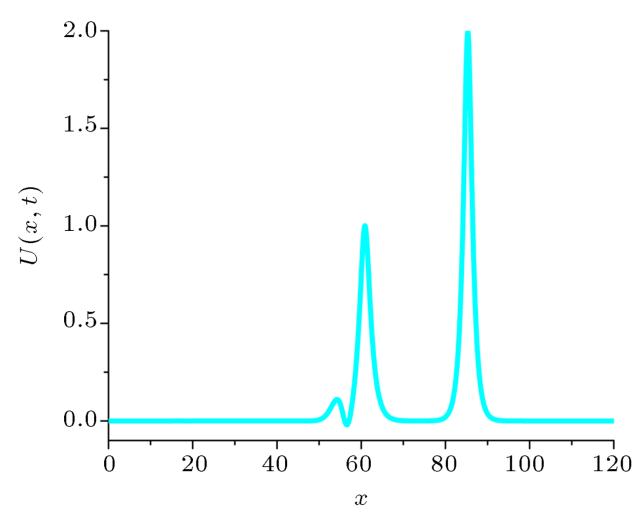

(d) $\begin{aligned} & x \\ & t=6\end{aligned}$

Figure 2. The collision of two solitary waves at $p=3$.

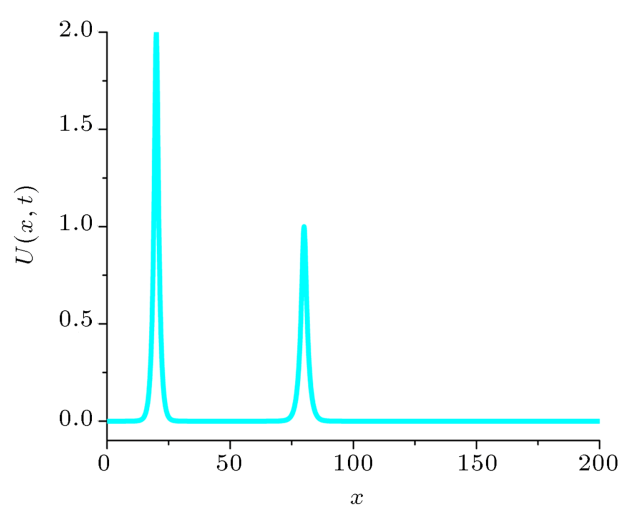

(a) $t=0$

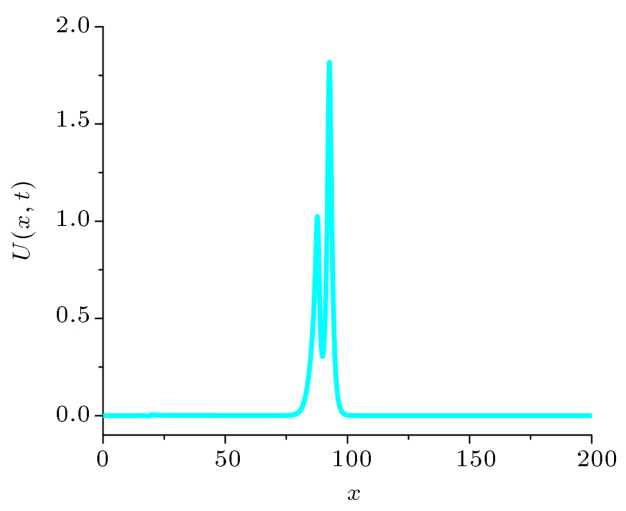

(c) $t=4$

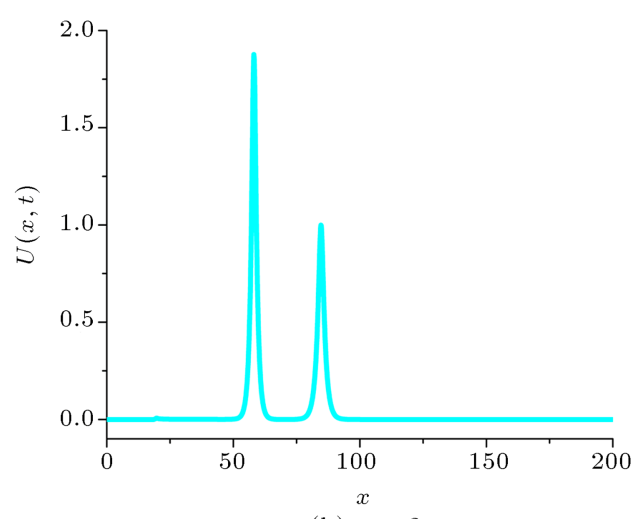

(b) $t=2$

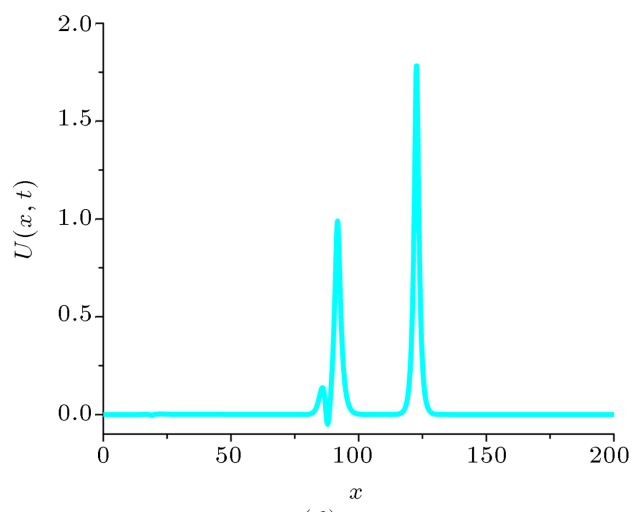

(d) $t=6$

Figure 3. The collision of two solitary waves at $p=4$. 


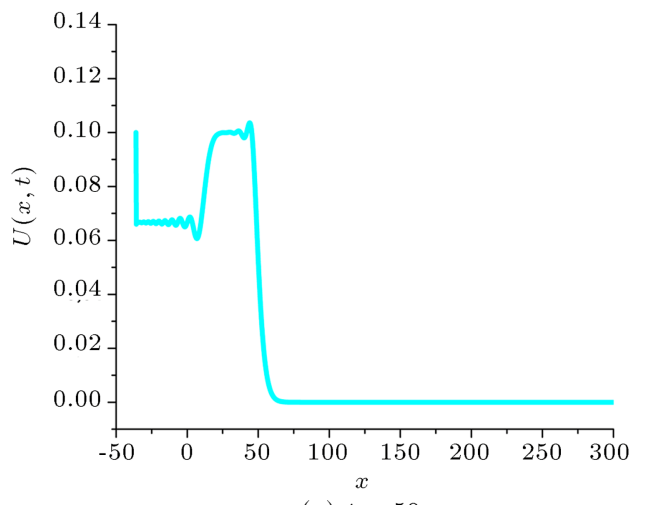

(a) $t=50$

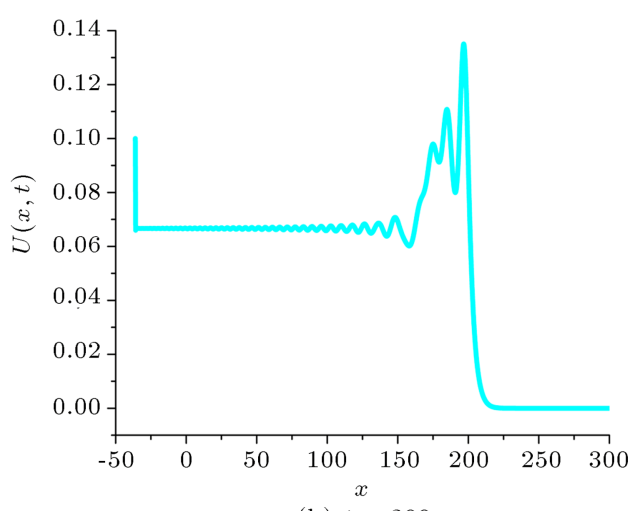

(b) $t=200$

Figure 4. The profile of the growth of an undular bore at $p=2$.

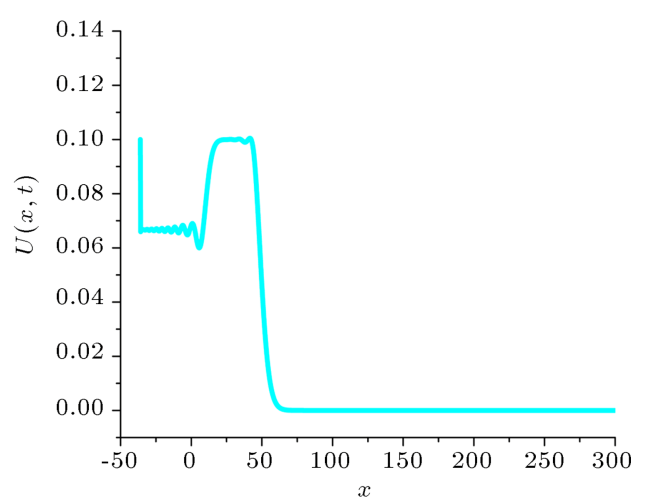

(a) $t=50$

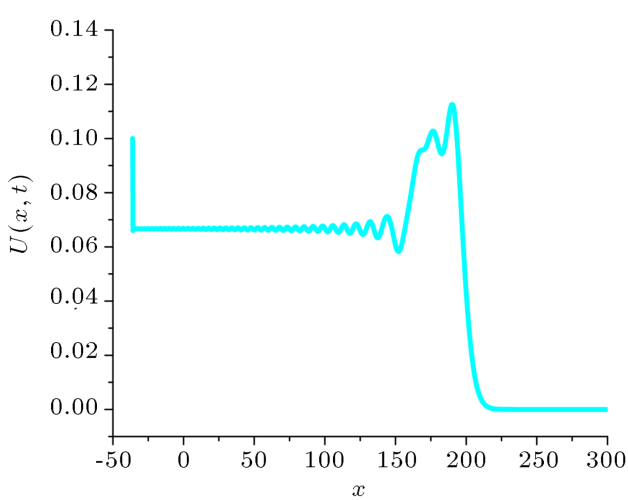

(b) $t=200$

Figure 5. The profile of growth of an undular bore at $p=3$.

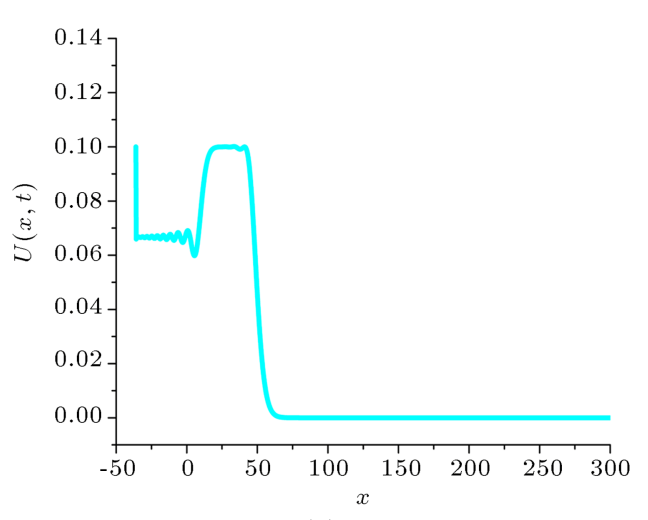

(a) $t=50$

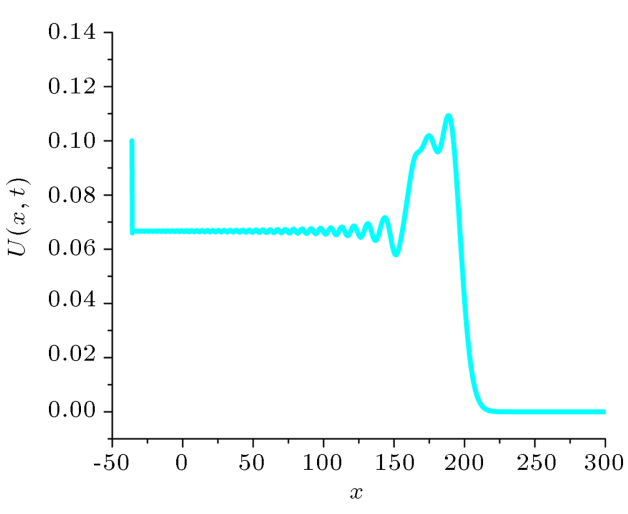

(b) $t=200$

Figure 6. The profile of the growth of an undular bore at $p=4$.

constructed for obtaining a numerical solution to the GRLW equation. Using the von-Neumann technique, the method was shown to be unconditionally stable. The QBSCM was tested with three examples including a single solitary wave, the collision of two solitary waves, and the growth of an undular bore. The error in $L_{2}$, and $L_{\infty}$ norms and three conservative quantities $I_{1}$, $I_{2}$, and $I_{3}$ were calculated to confirm the performance of the numerical scheme. The major point of the QBSCM is that it reduces the problem into a system of first-order ordinary differential equations. Then, the system produces the recurrence relationship whose solutions can be found through the penta-diagonal system. Further to that, it is easy to apply the method to different values of $\varepsilon$ and $p$, which affect the nonlinear term, velocity, and initial condition. The findings prove that three physical quantities of motion remain constant during wave propagation, and the results are the same as those of previous studies. The magnitude of the obtained error norms is adequately small, and it is better than the ones in earlier works. Hereby, the proposed scheme is a practical, accurate and powerful 
numerical technique. It can be confidingly used for solving similar types of nonlinear problems.

\section{Acknowledgements}

The authors are very grateful to anonymous referees for their detailed reading, precious comments, and proposals.

\section{References}

1. Peregrine, D.H. "Calculations of the development of an undular bore", Journal of Fluid Mechanics, 25, pp. 321-330 (1966).

2. Peregrine, D.H. "Long waves on a beach", Journal of Fluid Mechanics, 27, pp. 815-827 (1967).

3. Benjamin, T.B., Bona, J.L., and Mahony, J.J. "Model equations for long waves in non-linear dispersive systems", Philosophical Transactions of the Royal Society of London Series A, 272, pp. 47-78 (1972).

4. Morrison, P.J., Meiss, J.D., and Carey, J.R. "Scattering of RLW solitary waves", Physica D, 11, pp. 324-336 (1984).

5. Raslan, K.R. "Collocation method using quadratic Bspline for the RLW equation", International Journal of Computer Mathematics, 78, pp. 399-412 (2001).

6. Da ̆, I., Saka, B., and Irk, D. "Application of cubic Bsplines for numerical solution of the RLW equation", Applied Mathematics and Computation, 159(2), pp. 373-389 (2004).

7. Saka, B., Dă̆, I., and Irk, D. "Quintic B-spline collocation method for numerical solution of the RLW equation", The ANZIAM Journal, 49(3), pp. 389-410 (2008).

8. Saka, B., Sahin, A., and Dă̆, I. "B-spline collocation algorithms for numerical solution of the RLW equation", Numerical Methods for Partial Differential Equations, 27, pp. 581-607 (2011).

9. Soliman, A.A. and Hussien, M.H. "Collocation solution for RLW equation with septic spline", Applied Mathematics and Computation, 161(2), pp. 623-636 (2005).

10. Dă̆, I., Saka, B. and Irk, D. "Galerkin method for the numerical solution of the RLW equation using quintic B-splines", Journal of Computational and Applied Mathematics, 190, pp. 532-547 (2006).

11. Esen, A. and Kutluay, S. "Application of a lumped Galerkin method to the regularized long wave equation", Applied Mathematics and Computation, 174, pp. 833-845 (2006).

12. Mei, L. and Chen, Y. "Numerical solutions of RLW equation using Galerkin method with extrapolation techniques", Computer Physics Communications, 183, pp. 1609-1616 (2012).
13. Gardner, L.R.T., Gardner, G.A., Ayoub, F.A., and Amein, N.K. "Approximations of solitary waves of the MRLW equation by B-spline finite element", Arabian Journal for Science and Engineering, 22, pp. 183-193 (1997).

14. Haq, F., Islam, S., and Tirmizi, I.A. "A numerical technique for solution of the MRLW equation using quartic B-splines", Applied Mathematical Modelling, 34(12), pp. 4151-4160 (2010).

15. Karakoç, S.B.G., Yağmurlu, N.M., and Ucar, Y. "Numerical approximation to a solution of the modified regularized long wave equation using quintic Bsplines", Boundary Value Problems, 2013, pp. 1-17 (2013).

16. Karakoç, S.B.G., Ak, T., and Zeybek, H. "An efficient approach to numerical study of the MRLW equation with B-spline collocation method", Abstract and Applied Analysis, 2014, pp. 1-15 (2014).

17. Khalifa, A.K., Raslan, K.R., and Alzubaidi, H.M. "A collocation method with cubic B-splines for solving the MRLW equation", Journal of Computational and Applied Mathematics, 212, pp. 406-418 (2008).

18. Raslan, K.R. and EL-Danaf, T.S. "Solitary waves solutions of the MRLW equation using quintic Bsplines", Journal of King Saud University - Science, 22(3), pp. 161-166 (2010).

19. Ali, A. "Mesh free collocation method for numerical solution of initial-boundary value problems using radial basis functions", Ph.D. Thesis, Ghulam Ishaq Khan Institute of Engineering Sciences and Technology, Pakistan (2009).

20. Dağ, I., Irk, D., and Sari, M. "The extended cubic Bspline algorithm for a modified regularized long wave equation", Chinese Physics B, 22(4), pp. 1-6 (2013).

21. Abo Essa, Y.M., Abouefarag, I., and Rahmo, E.-D. "The numerical solution of the MRLW equation using the multigrid method", Applied Mathematics, 5, pp. 3328-3334 (2014).

22. Bona, J.L., McKinney, W.R., and Restrepo, J.M. "Stable and unstable solitary-wave solutions of the generalized regularized long-wave equation", Journal of Nonlinear Science, 10, pp. 603-638 (2000).

23. Hammad, D.A. and El-Azab, M.S. "A $2 \mathrm{~N}$ order compact finite difference method for solving the generalized regularized long wave (GRLW) equation", Applied Mathematics and Computation, 253, pp. 248261 (2015).

24. Huang, D.M. and Zhang, L.W. "Element-free approximation of generalized regularized long wave equation", Mathematical Problems in Engineering, 2014, pp. 1-10 (2014).

25. Mokhtari, R. and Mohammadi, M. "Numerical solution of GRLW equation using Sinc-collocation method", Computer Physics Communications, 181, pp. 1266-1274 (2010). 
26. Roshan, T. "A Petrov-Galerkin method for solving the generalized regularized long wave (GRLW) equation", Computers and Mathematics with Applications, 63, pp. 943-956 (2012).

27. Soliman, A.A. "Numerical simulation of the generalized regularized long wave equation by He's variational iteration method", Mathematics and Computers in Simulation, 70, pp. 119-124 (2005).

28. Zhang, L. "A finite difference scheme for generalized regularized long-wave equation", Applied Mathematics and Computation, 168, pp. 962-972 (2005).

29. Kaya, D. and El-Sayed, S.M. "An application of the decomposition method for the generalized $\mathrm{KdV}$ and RLW equations", Chaos, Solitons and Fractals, 17, pp. 869-877 (2003).

30. Hamdi, S., Enright, W.H., Schiesser, W.E., and Gottlieb, J.J. "Exact solutions and invariants of motion for general types of regularized long wave equations", Mathematics and Computers in Simulation, 65, pp. 535-545 (2004).

31. Ramos, J.I. "Solitary wave interactions of the GRLW equation", Chaos, Solitons \& Fractals, 33, pp. 479-491 (2007).

32. Mohammadi, R. "Exponential B-spline collocation method for numerical solution of the generalized regularized long wave equation", Chinese Physics B, 24, pp. 1-14 (2015).

33. Zeybek, H. and Karakoç, S.B.G. "A numerical investigation of the GRLW equation using lumped Galerkin approach with cubic B-spline", SpringerPlus, 5, pp. 1-17 (2016).

34. Karakoç, S.B.G. and Zeybek, H. "Solitary-wave solutions of the GRLW equation using septic B-spline collocation method", Applied Mathematics and Computation, 289, pp. 159-171 (2016).

35. Irk, D. and Dağ, I. "Quintic B-spline collocation method for the generalized nonlinear Schrödinger equation", Journal of the Franklin Institute, 348, pp. 378-392 (2011).
36. Ismail, M.S. "Numerical solution of complex modified Korteweg-de Vries equation by collocation method", Communications in Nonlinear Science and Numerical Simulation, 14, pp. 749-759 (2009).

37. Mittal, R.C. and Tripathi, A. "Numerical solutions of generalized Burgers-Fisher and generalized BurgersHuxley equations using collocation of cubic B-splines", International Journal of Computer Mathematics, 93, pp. 1053-1077 (2015).

38. Ak, T., Karakoc, S.B.G., and Biswas, A. "Application of Petrov-Galerkin finite element method to shallow water waves model: modified Korteweg-de Vries equation", Scientia Iranica, 24, pp. 1148-1159 (2017).

39. Prenter, P.M., Splines and Variational Methods, J. Wiley, New York (1975).

40. Rubin, S.G. and Graves, R.A., A Cubic Spline Approximation for Problems in Fluid Mechanics, NASA TR R-436, Washington, DC (1975).

\section{Biographies}

Halil Zeybek graduated from Ondokuz Mayıs University in 2008 with a BSc degree in Mathematics. He received his MSc and $\mathrm{PhD}$ degrees in Applied Mathematics from Nevşehir Hacı Bektaş Veli University in 2011 and 2016, respectively. He is currently a Research Assistant in Abdullah Gul University. He has done his research and publications in the areas of solitary waves, fluid dynamics, numerical analysis, and applied mathematics.

Seydi Battal Gazi Karakoç graduated from Selçuk University in 2001 with a BSc degree in Mathematics. $\mathrm{He}$ received his $\mathrm{MSc}$ and $\mathrm{PhD}$ degrees in Applied Mathematics from İnönü University in 2006 and 2011, respectively. He is currently an Assistant Professor in Nevşehir Hacı Bektaş Veli University. He has done his research and publications in the areas of finite element method, numerical simulation, and applied mathematics. 